\title{
Form factor expansions in the 2D Ising model and Painlevé VI
}

\author{
Vladimir V. Mangazeev ${ }^{1}$ \\ Department of Theoretical Physics, \\ Research School of Physics and Engineering, \\ Australian National University, Canberra, ACT 0200, Australia \\ Anthony J. Guttmann² \\ ARC Centre of Excellence for \\ Mathematics and Statistics of Complex Systems, \\ Department of Mathematics and Statistics, \\ The University of Melbourne, Victoria 3010, Australia
}

\begin{abstract}
We derive a Toda-type recurrence relation, in both high and low temperature regimes, for the $\lambda$ - extended diagonal correlation functions $C(N, N ; \lambda)$ of the two-dimensional Ising model, using an earlier connection between diagonal form factor expansions and tau-functions within Painlevé VI (PVI) theory, originally discovered by Jimbo and Miwa. This greatly simplifies the calculation of the diagonal correlation functions, particularly their $\lambda$-extended counterparts.

We also conjecture a closed form expression for the simplest off-diagonal case $C^{ \pm}(0,1 ; \lambda)$ where a connection to PVI theory is not known. Combined with the results for diagonal correlations these give all the initial conditions required for the $\lambda$-extended version of quadratic difference equations for the correlation functions discovered by McCoy, Perk and Wu. The results obtained here should provide a further potential algorithmic improvement in the $\lambda$-extended case, and facilitate other developments.
\end{abstract}

\footnotetext{
${ }^{1}$ email: Vladimir.Mangazeev@anu.edu.au

${ }^{2}$ email: tonyg@ms.unimelb.edu.au
} 


\section{Introduction}

The 2D Ising model in zero magnetic field is arguably the most important solvable model in theoretical statistical mechanics. Its free energy [1] and the spontaneous magnetization $[2,3]$ were computed a long time ago by Onsager and Yang.

Despite more than half a century of attempts by many outstanding scientists, a closed form expression for the susceptibility is still unknown. We have a vast amount of numerical and analytical information, mostly derived from careful analysis of exact series expansions. The best algorithm for such series expansions, due to Orrick, Nickel, Guttmann and Perk [4], is based on expressing the susceptibility in terms of correlation functions, and using quadratic difference equations, due to McCoy, Perk and Wu [5], [6], [7] to recursively calculate the required correlation functions. A related study that gives complementary analytic information is based on the multiparticle expansion [8] of the susceptibility derived by Wu, McCoy Tracy and Barouch in 1976. In either formulation, one requires the correlation functions or their $\lambda$ - extended counterparts (see (7-8) below).

In this paper we present a different method for the calculation of diagonal pair correlation functions and form factor expansions that occur in the theory of the $2 \mathrm{D}$ Ising model in zero magnetic field.

We shall restrict ourselves to the case of the symmetric 2D Ising model defined by the interaction energy

$$
E=-J \sum_{i, j}\left(\sigma_{i, j} \sigma_{i, j+1}+\sigma_{i, j} \sigma_{i+1, j}\right)
$$

where $J>0$ (ferromagnetic regime) and spins $\sigma_{i, j}= \pm 1$ are assigned to each site of a square lattice.

The $2 \mathrm{D}$ Ising model has a second order phase transition at the critical temperature $T_{c}$ defined by the condition

$$
\sinh \left(2 J / k_{B} T_{c}\right)=1,
$$

where $k_{B}$ is Boltzmann's constant.

A study of the pair correlation functions

$$
C(M, N)=\left\langle\sigma_{0,0} \sigma_{M, N}\right\rangle
$$

started long ago [9] when they were expressed in terms of determinants [10,11]. Subsequent studies of correlation functions $[7,8,12-17]$ led to infinite form factor expansions with every form factor given by a multiple integral [8].

Note also that there are two special cases where a simpler determinant representation for correlation functions is known. These are the cases of row and diagonal correlations $M=0$ and $M=N$ respectively, for any $N$. In both cases there exists a representation for pair correlation functions by $N \times N$ Toeplitz determinants [11].

In 1980 Jimbo and Miwa [18] found yet another approach to the calculation of the diagonal correlations $C(N, N)$. They showed that $C(N, N)$ satisfies the "sigma" form of the Painlevé VI equation and produced a system of recurrence relations which allows one to calculate diagonal correlations $C(N+1, N+1)$ in terms of $C(N, N)$ and $C^{*}(N, N)$ which are the correlation functions obtained by the duality transformation interchanging high/low temperature regimes.

Moreover, Jimbo and Miwa introduced the so-called $\lambda$-extension (or isomonodromic deformation) of the correlation functions using a multi-particle expansions in terms of free fermion 
operators. Then they related this to the matrix Schlesinger equations with integrability conditions given by the Painlevé VI equation. ${ }^{1}$ In such an approach the parameter $\lambda$ describes monodromy properties of solutions for Schlesinger equations and plays the role of boundary conditions for the Painlevé VI equation.

Similar $\lambda$-extensions naturally appear in the Fredholm determinant approach [8] and can be regarded as generating functions for the form factors (see (7-8) below). The case $\lambda=1$ corresponds to the symmetric Ising model.

Starting with the asymptotics of diagonal correlations in the high/low temperature regimes, the authors of [20] studied series solutions of the Painlevé VI equation and showed the existence of the one-parameter extension of $C(N, N)$ which exactly matches the form factor extension. They conjectured that the Jimbo-Miwa $\lambda$-extension and the form factor $\lambda$-extension are actually the same, with the parameter $\lambda$ in (7-8) playing the role of boundary condition for the Painlevé VI equation.

We note that such an identification is a result of experimental mathematics, confirmed by a large amount of computer calculation. In this paper we will rely on this, even though a rigorous mathematical proof of such an identification is still missing.

A different approach to calculation of correlation functions was developed by McCoy and $\mathrm{Wu}$ [5] and by Perk [7] in 1980. They discovered a set of quadratic difference equations satisfied by the pair correlation functions of the $2 \mathrm{D}$ Ising model. These equations were developed further in $[21]$.

In the symmetric case the quadratic difference equations allow one to calculate all correlation functions recursively, once we know the "initial conditions": diagonal correlations $C(N, N)$ and $C^{*}(N, N)$ together with the nearest-neighbor correlations $C(0,1)$ and $C^{*}(0,1)$ [22]. The diagonal correlations can be calculated from the Jimbo-Miwa recurrence relations and when combined with well known expressions for the nearest-neighbor correlations, gives a very effective method for series calculations of the correlation functions and the susceptibility [4].

In this paper we present a different method of calculation of diagonal correlations in the $\lambda$-extended case. It is based on the explicit relationship of such correlations to tau-functions in Painlevé VI theory. We also conjecture a formula for the $\lambda$-extension of the nearest neighbor correlations $C(0,1)$. The series in the elliptic nome for the form factors contributing to $C(0,1)$ were first conjectured in sec. 5.2 of [4]. At that time it was not clear how to sum up such expressions $^{2}$. Here we have used a completely different method to obtain a closed form expression for $C(0,1)$ in terms of elliptic functions.

Following [24] we will use two variables $s$ and $t$;

$$
t=s^{4}, \quad s= \begin{cases}\sinh \left(2 E / k_{B} T\right), & T>T_{c}, \\ \sinh \left(2 E / k_{B} T\right)^{-1}, & T<T_{c} .\end{cases}
$$

Let us introduce a special notation for diagonal correlation functions

$$
C_{N}^{ \pm}(t)=C(N, N)
$$

where \pm denotes $T \gtrless T_{c}$ regimes, respectively. We note in the symmetric case the dual of $C_{N}^{ \pm}(t)$ is $C_{N}^{\mp}(t)$.

\footnotetext{
${ }^{1}$ The idea of a $\lambda$-extension was made even earlier in McCoy, Tracy and $\mathrm{Wu}[19]$ in connection with solutions to Painlevé III.

${ }^{2}$ After this paper appeared, the technique for summing up such series was given by McCoy et al. in [23].
} 
Our main result is the following Toda-type relation for diagonal correlations

$$
t \frac{d^{2}}{d t^{2}} \log C_{N}^{ \pm}(t)+\frac{d}{d t} \log C_{N}^{ \pm}(t)+\frac{N^{2}}{(1-t)^{2}}=\frac{\left(N^{2}-\frac{1}{4}\right)}{(1-t)^{2}} \frac{C_{N+1}^{ \pm}(t) C_{N-1}^{ \pm}(t)}{\left(C_{N}^{ \pm}(t)\right)^{2}},
$$

valid for both temperature regimes at $N=1,2, \ldots$. Supplemented by initial conditions for $N=0,1$, relation (6) gives a very effective way to calculate $C_{N}^{ \pm}(t)$ for $N=2,3,4, \ldots$ It took us under 10 mins to calculate $C_{N}^{+}(t)$ for $N=2, \ldots, 20$ on a laptop computer using a Mathematica program.

What's more important is that eqn (6) remains valid for the $\lambda$-extension of diagonal correlation functions. In fact, as shown in section 5 , a form factor expansion for $N=0$ is sufficient to calculate the generalized diagonal correlations for $N=1,2, \ldots$.

The paper is organized as follows. In section 2 we define the $\lambda$ - extended pair correlation functions, give integral formulae for the form factors and recall the result of Jimbo and Miwa on diagonal correlators. In section 3 we list some basic facts on the Hamiltonian formulation of Painlevé VI theory and prove a Toda-type relation for tau-functions related by a special shift transformation. In section 4 we establish a connection with the Jimbo-Miwa result and prove relation (6). In section 5 we calculate generalized diagonal correlators at arbitrary $\lambda$ for $N=0,1,2$. In section 6 we present a closed form expression for the $\lambda$ - extension of the first nontrivial off-diagonal correlation function $M=0, N=1$. Together with the results of Section 4, these provide all initial conditions required for the quadratic difference equation satisfied by the $\lambda$-extended correlation functions, and so allows, in principle, the rapid calculation of any required correlation function. The major advance is in the calculation of $\lambda$-extended correlation functions. In section 7 we discuss the results obtained, and mention possible further developments.

\section{Form factor expansions of pair correlation functions}

We start with the $\lambda$ - expansion of pair correlation functions in multi-particle components

$$
C^{+}(M, N ; \lambda)=s^{-1}(1-t)^{1 / 4} \sum_{n=0}^{\infty} \lambda^{2 n+1} \hat{C}^{(2 n+1)}(M, N), \quad T>T_{c},
$$

and

$$
C^{-}(M, N ; \lambda)=(1-t)^{1 / 4}\left(1+\sum_{n=1}^{\infty} \lambda^{2 n} \hat{C}^{(2 n)}(M, N)\right), \quad T<T_{c},
$$

where for $n>0$ (see, for example, formula (4.2) in [4], given here for the symmetric case)

$$
\begin{gathered}
\hat{C}^{(n)}(M, N)=\frac{1}{n !} \int_{-\pi}^{\pi} \frac{d \omega_{1}}{2 \pi} \ldots \int_{-\pi}^{\pi} \frac{d \omega_{n}}{2 \pi}\left[\prod_{i=1}^{n} \frac{x_{i}^{M}}{\sinh \gamma_{i}}\right]\left[\prod_{1 \leq i<j \leq n} h_{i j}\right]^{2} \cos \left(N \sum_{i=1}^{n} \omega_{i}\right), \\
\sinh \gamma_{i}=\left(y_{i}^{2}-1\right)^{1 / 2}, \quad x_{i}=y_{i}-\left(y_{i}^{2}-1\right)^{1 / 2}, \quad y_{i}=s+s^{-1}-\cos \omega_{i}, \\
h_{i j}=\frac{2\left(x_{i} x_{j}\right)^{1 / 2} \sin \left(\left(\omega_{i}-\omega_{j}\right) / 2\right)}{1-x_{i} x_{j}} .
\end{gathered}
$$

We shall call $C^{ \pm}(M, N ; \lambda)$ generalized correlation functions. The pair correlations for the symmetric Ising model are obtained by setting $\lambda=1$

$$
C^{ \pm}(M, N)=C^{ \pm}(M, N ; 1)
$$


Using a different approach Jimbo and Miwa [18] in 1980 introduced the function

$$
\sigma_{N}(t)= \begin{cases}t(t-1) \frac{d}{d t} \log C^{+}(N, N)-\frac{1}{4}, & T>T_{c}, \\ t(t-1) \frac{d}{d t} \log C^{-}(N, N)-\frac{t}{4}, & T<T_{c} .\end{cases}
$$

and showed that it satisfies the "sigma" form of Painlevé VI

$$
\left(t(t-1) \frac{d^{2} \sigma}{d t^{2}}\right)^{2}+4 \frac{d \sigma}{d t}\left((t-1) \frac{d \sigma}{d t}-\sigma-\frac{1}{4}\right)\left(t \frac{d \sigma}{d t}-\sigma\right)=N^{2}\left((t-1) \frac{d \sigma}{d t}-\sigma\right)^{2} .
$$

In 2004 [25] Forrester and Witte gave a new derivation of this result working from the Toeplitz determinant form rather than the Fredholm form.

As mentioned in the introduction, Jimbo and Miwa also considered an isomonodromic $\lambda$ extension of $C^{ \pm}(N, N)$ which also satisfies (14). Using extensive computer calculations Boukraa et al. [20] observed that the Jimbo-Miwa $\lambda$-extension is the same as the form factor expansions (7-8). It provides an opportunity to investigate properties of generalized diagonal correlation functions $C^{ \pm}(N, N ; \lambda)$ by studying solutions of (14).

In the rest of the paper we will assume $\lambda$ to be arbitrary unless its value is explicitly specified. We shall also use the same notation introduced in (5) for generalized diagonal correlation functions (7-8)

$$
C_{N}^{ \pm}(t) \equiv C^{ \pm}(N, N ; \lambda)
$$

and simply omit the dependence on $\lambda$.

The solutions of (14) can be specified by their expansion near $t \rightarrow 0$. In [20] Boukraa et al. analyzed series expansions of $C_{N}^{ \pm}(t)$ at $t \rightarrow 0$ and demonstrated that

$$
C_{N}^{-}(t)=(1-t)^{1 / 4}+\lambda^{2} \frac{(1 / 2)_{N}(3 / 2)_{N}}{4((N+1) !)^{2}} t^{N+1}(1+O(t))
$$

and

$$
C_{N}^{+}(t)=\lambda(1-t)^{1 / 4} f_{N, N}^{(1)}+\lambda^{3} \frac{(1 / 2)_{N}\left((3 / 2)_{N}\right)^{2}}{16(N+1) !(N+2) !} t^{3 N / 2+2}(1+O(t))
$$

where

$$
f_{N, N}^{(1)}=t^{N / 2} \frac{(1 / 2)_{N}}{N !}{ }_{2} F_{1}\left(\frac{1}{2}, N+\frac{1}{2}, N+1 ; t\right),
$$

and $(a)_{N}=\Gamma(a+N) / \Gamma(a)$ is the usual Pochhammer symbol. We shall use (16-17) to fix a normalization of recurrence relations in section 4 .

\section{Some facts from Painlevé VI theory}

In this section we shall briefly review the properties of the Hamiltonian form of the Painlevé VI $\left(\mathbf{P}_{V I}\right)$ equation [26,27], given by eqn $(22)$. Our main reference is the work of Okamoto [28].

For each solution of the $\mathbf{P}_{V I}$ equation, one can construct a Hamiltonian function $H(t) \equiv$ $H(t ; \mathbf{b})$ which depends on four parameters

$$
\mathbf{b}=\left(b_{1}, b_{2}, b_{3}, b_{4}\right) .
$$

A tau-function $\tau(t) \equiv \tau(t ; \mathbf{b})$ related to the Hamiltonian $H(t ; \mathbf{b})$ is defined by

$$
H(t ; \mathbf{b})=\frac{d}{d t} \log \tau(t ; \mathbf{b}) .
$$


Let us introduce an auxiliary Hamiltonian $h(t) \equiv h(t ; \mathbf{b})$,

$$
h(t)=t(t-1) H(t)+e_{2}\left(b_{1}, b_{3}, b_{4}\right) t-\frac{1}{2} e_{2}\left(b_{1}, b_{2}, b_{3}, b_{4}\right)
$$

where $e_{i}\left(x_{1}, \ldots, x_{n}\right)$ is the $i$-th elementary symmetric function in $n$ variables and a set of $x_{i}$ 's can be a subset of $b_{i}$ 's as in (21).

The function $h(t)$ solves the equation $\mathbf{E}_{V I}[\mathbf{b}]^{3}$

$$
h^{\prime}(t)\left[t(1-t) h^{\prime \prime}(t)\right]^{2}+\left[h^{\prime}(t)\left[2 h(t)-(2 t-1) h^{\prime}(t)\right]+b_{1} b_{2} b_{3} b_{4}\right]^{2}=\prod_{k=1}^{4}\left(h^{\prime}(t)+b_{k}^{2}\right) .
$$

The group $G$ of Backlund transformations of $\mathbf{P}_{V I}$ is isomorphic to the affine Weyl group of type $F_{4}: W_{a}\left(F_{4}\right)$. It contains the following transformations of parameters (not all of them are independent)

$$
\begin{gathered}
w_{1}: b_{1} \leftrightarrow b_{2}, \quad w_{2}: b_{2} \leftrightarrow b_{3}, \quad w_{3}: b_{3} \leftrightarrow b_{4}, \quad w_{4}: b_{3} \rightarrow-b_{3}, b_{4} \rightarrow-b_{4}, \\
x^{1}: \kappa_{0} \leftrightarrow \kappa_{1}, \quad x^{2}: \kappa_{0} \leftrightarrow \kappa_{\infty}, \quad x^{3}: \kappa_{0} \leftrightarrow \theta
\end{gathered}
$$

where

$$
\kappa_{0}=b_{1}+b_{2}, \quad \kappa_{1}=b_{1}-b_{2}, \quad \kappa_{\infty}=b_{3}-b_{4}, \quad \theta=b_{3}+b_{4}+1
$$

and the parallel transformation

$$
l_{3}: \mathbf{b} \equiv\left(b_{1}, b_{2}, b_{3}, b_{4}\right) \rightarrow \mathbf{b}^{+} \equiv\left(b_{1}, b_{2}, b_{3}+1, b_{4}\right) .
$$

For each transformation $s$ from the group $W_{a}\left(F_{4}\right)$ such that

$$
s: \mathbf{b} \mapsto \mathbf{b}_{\mathbf{s}}
$$

one can construct another auxiliary Hamiltonian $h\left(t ; \mathbf{b}_{\mathbf{s}}\right)$ which satisfies $\mathbf{E}_{V I}\left[\mathbf{b}_{\mathbf{s}}\right]$. The function $h\left(t ; \mathbf{b}_{\mathbf{s}}\right)$ is a rational function of $t$ and

$$
\left\{h(t ; \mathbf{b}), \frac{d}{d t} h(t ; \mathbf{b}), \frac{d^{2}}{d t^{2}} h(t ; \mathbf{b})\right\} .
$$

If we consider a sequence of transformations related to $l_{3}^{m}, m=0, \pm 1, \pm 2, \ldots$ and construct an associated sequence $h_{m}(t)$ and related tau-functions $\tau_{m}(t)$, then it is well known [28] that such tau-functions satisfy Toda-type recurrence relations.

Let us consider a different transformation $l_{s} \in G$ :

$$
l_{s}=x_{1} x_{2} l_{3} x_{2} x_{1}
$$

where the action of the $x_{i}$ 's and $l_{3}$ on $b_{i}$ 's is defined by (23-26).

After simple calculations one obtains

$$
l_{s}^{ \pm 1}:\left(b_{1}, b_{2}, b_{3}, b_{4}\right) \mapsto\left(b_{1} \pm \frac{1}{2}, b_{2} \mp \frac{1}{2}, b_{3} \pm \frac{1}{2}, b_{4} \pm \frac{1}{2}\right) .
$$

To calculate the expressions for related auxiliary Hamiltonians we need to use the results from Okamoto's paper [28], sections 2,3. Here we shall only present the answer

\footnotetext{
${ }^{3}$ So named by Okamoto [28] in his studies of PVI. It is in fact a generalization of the "sigma" form of PVI (14).
} 


$$
h_{ \pm}(t) \equiv h\left(t, \mathbf{b}^{ \pm}\right)=h(t)-\frac{1}{4} \pm \frac{\tilde{S}_{1}}{4}+\frac{2 t(t-1)^{2} h^{\prime \prime}(t) \pm\left[2 \tilde{S}_{1} h(t)+2 \tilde{S}_{3}(1-t)-\tilde{S}_{1} \tilde{S}_{2}\right]}{2\left(2(t-1) h^{\prime}(t)-2 h(t)+\tilde{S}_{2}\right)}
$$

where

$$
\tilde{S}_{i}=e_{i}\left(b_{1},-b_{2}, b_{3}, b_{4}\right), \quad \mathbf{b}^{ \pm} \equiv l_{s}^{ \pm 1}(\mathbf{b})=\left(b_{1} \pm \frac{1}{2}, b_{2} \mp \frac{1}{2}, b_{3} \pm \frac{1}{2}, b_{4} \pm \frac{1}{2}\right) .
$$

Now we can follow the Okamoto derivation of Toda relations for tau-functions. Using (21) and (31) we obtain

$$
\begin{aligned}
& h_{+}(t)+h_{-}(t)-2 h(t)=t(t-1)\left[H_{+}(t)+H_{-}(t)-2 H(t)+\frac{3 t}{2}\right]= \\
& =-\frac{t}{2}+\frac{t(t-1)^{2} h^{\prime \prime}(t)}{2(t-1) h^{\prime}(t)-2 h(t)+\tilde{S}_{2}} .
\end{aligned}
$$

Substituting

$$
H_{ \pm}(t)=\frac{d}{d t} \log \left[\tau_{ \pm}(t)\right], \quad H(t)=\frac{d}{d t} \log [\tau(t)]
$$

and integrating over $t$ we get

$$
B(t-1)^{2} \frac{\tau_{+}(t) \tau_{-}(t)}{\tau^{2}(t)}=(t-1) h^{\prime}(t)-h(t)+\frac{\tilde{S}_{2}}{2},
$$

or after using the expression for $h(t)$ in terms of $\tau(t)$,

$$
t \frac{d^{2}}{d t^{2}} \log \tau(t)+\frac{d}{d t} \log \tau(t)=B \frac{\tau_{+}(t) \tau_{-}(t)}{\tau^{2}(t)},
$$

where $B$ is the integration constant.

We have just shown that if we introduce a sequence of parameters

$$
\mathbf{b}_{N} \equiv\left(b_{1}+\frac{N}{2}, b_{2}-\frac{N}{2}, b_{3}+\frac{N}{2}, b_{4}+\frac{N}{2}\right)
$$

and associated sequences of Hamiltonians and tau-functions

$$
h_{N}(t)=h\left(t, \mathbf{b}_{N}\right), \quad H_{N}(t)=H\left(t, \mathbf{b}_{N}\right), \quad \tau_{N}(t)=\tau\left(t, \mathbf{b}_{N}\right),
$$

then tau-functions $\tau_{N}(t)$ satisfy Toda-type recurrence relations

$$
t \frac{d^{2}}{d t^{2}} \log \tau_{N}(t)+\frac{d}{d t} \log \tau_{N}(t)=B_{N} \frac{\tau_{N+1}(t) \tau_{N-1}(t)}{\tau^{2}(t)}
$$

where the normalization constant $B_{N}$ can depend on the parameters $b_{1}, b_{2}, b_{3}, b_{4}$ and $N$. 


\section{Recurrence relations for $C_{N}^{ \pm}(t)$}

Now we use the results from the previous section to obtain recurrence relations for generalized correlation functions $C_{N}^{ \pm}(t)$ valid for arbitrary $\lambda$.

First we need to establish a connection between equations (14) and (22). Let us consider the equation $\mathbf{E}_{V I}\left[\mathbf{b}_{N}\right]$ (22) with parameters (37) and choose

$$
b_{1}=-1 / 2, \quad b_{2}=-1 / 2, \quad b_{3}=0, \quad b_{4}=0 .
$$

If we denote a corresponding solution of $(22)$ as $h_{N}(t)$, then after a substitution

$$
h_{N}(t)=\sigma_{N}(t)-\frac{N^{2}}{4} t+\frac{1}{8}
$$

equation (22) coincides with (14).

Now combining formulae (13, 20-21) and (41) we can find a connection between tau-functions $\tau_{N}(t)$ from the previous section and $C_{N}^{ \pm}(t)$, viz.

$$
\tau_{N}(t)= \begin{cases}C_{N}^{+}(t)(1-t)^{-N^{2}} t^{\frac{N}{2}}, & T>T_{c} \\ C_{N}^{-}(t)(1-t)^{-N^{2}} t^{\frac{N}{2}-\frac{1}{4}}, & T<T_{c}\end{cases}
$$

Substituting (42) into (39) we finally arrive at recurrence relations for generalized diagonal correlation functions $C_{N}^{ \pm}(t)$,

$$
t \frac{d^{2}}{d t^{2}} \log C_{N}^{ \pm}(t)+\frac{d}{d t} \log C_{N}^{ \pm}(t)+\frac{N^{2}}{(1-t)^{2}}=\frac{\left(N^{2}-\frac{1}{4}\right)}{(1-t)^{2}} \frac{C_{N+1}^{ \pm}(t) C_{N-1}^{ \pm}(t)}{\left(C_{N}^{ \pm}(t)\right)^{2}}
$$

The normalization constant $B_{N}$ in (39) can be calculated by substituting expansions (16-17) at $t \rightarrow 0$ into (43). It appears that

$$
B_{N}=N^{2}-\frac{1}{4}
$$

for both the $T>T_{c}$ and $T<T_{c}$ regimes.

Let us comment on these Toda-type relations for $C_{N}^{ \pm}(t)$ which are valid for arbitrary values of $\lambda$. They allow us to calculate diagonal correlations for any $N=2,3,4, \ldots$ starting with $C_{0}(t)$ and $C_{1}(t)$. We don't need to use correlations at the dual temperature $s^{-1}$ as in [18]. In fact, when $\lambda$ is arbitrary, it is sufficient to know $C_{0}^{ \pm}(t)$ only, as $C_{1}^{ \pm}(t)$ can then be calculated from (43) as we shall see in the next section.

To our knowledge, equations (43) have not previously appeared in the literature. They provide a very efficient method for the calculation of diagonal form-factors and sums in formulae (7-8) in closed form.

\section{The Picard solution and calculation of $C_{0}^{ \pm}(t)$ and $C_{1}^{ \pm}(t)$}

In this section we shall calculate initial conditions for the recursion (43). The functions $C_{0}^{ \pm}(t)$ are simply related to tau-functions $\tau_{0}(t)$ by (42). A related function $h_{0}(t)$, given by eqn (41) solves the equation $\mathbf{E}_{V I}[\mathbf{b}]$ (22) with parameters $b_{i}$ given by eqn (40). It is well known that a general solution of (22) depending on two arbitrary parameters (specified by initial conditions) can be found in this case. 
Consider another set of parameters $b_{i}$

$$
b_{1}=0, \quad b_{2}=0, \quad b_{3}=-\frac{1}{2}, \quad b_{4}=-\frac{1}{2} .
$$

This choice corresponds to the Picard solution [29] of the Painlevé VI equation which can be found in closed form (see, for example, [30]) and expressed in terms of the elliptic Weierstrass function depending on two arbitrary parameters.

One can represent solutions of Painlevé equations in terms of logarithmic derivatives of ratios of functions with no movable singularities. As one of many examples of such a representation, we mention a representation of solutions of the $\mathbf{P}_{V I}$ as logarithmic derivatives of tau-functions (see section 4.3 of [28]).

A location of zeros of tau-functions for the Picard solution (and related the Hitchin solution) has been investigated by Kitaev and Korotkin [31] and recently by Brezhnev [32]. It follows that the tau-function for the Picard case should be proportional to the Jacobi theta-function depending on parameters specified by initial conditions. A detailed calculation of tau-functions for the Picard class solutions has been given recently by the first author in [33]. Here we will use these results.

First, let us denote an auxiliary Hamiltonian and related tau-function for the Picard case (45) as $h_{P}(t)$ and $\tau_{P}(t)$ respectively. Since the equation (22) is symmetric in the $b_{i}$ 's we immediately conclude that

$$
h_{0}(t)=h_{P}(t) .
$$

Using (21) and integrating over $t$ we produce a relation between tau-functions

$$
\tau_{0}(t)=(1-t)^{1 / 4} \tau_{P}(t)
$$

We introduce an elliptic parameterization and define the elliptic modulus $k$ as

$$
k=s^{2}, \quad t=k^{2} .
$$

Following [34] we will define theta functions $\theta_{i}(x \mid \tau)$ with quasi-periods $\pi$ and $\pi \tau$. The elliptic modulus $k$ in (48) and its complement $k^{\prime}$ are

$$
k=\frac{\theta_{2}(0 \mid \tau)^{2}}{\theta_{3}(0 \mid \tau)^{2}}, \quad k^{\prime}=\frac{\theta_{4}(0 \mid \tau)^{2}}{\theta_{3}(0 \mid \tau)^{2}}, \quad k^{2}+k^{\prime 2}=1 .
$$

It will be more convenient to use the parameter $t$ as the second argument of the elliptic functions

$$
\tau=i \frac{\mathrm{K}^{\prime}(t)}{\mathrm{K}(t)}, \quad q=e^{i \pi \tau}, \quad \mathrm{K}(t)=\frac{\pi}{2} \theta_{3}^{2}(0 \mid \tau), \quad \mathrm{K}^{\prime}(t)=K(1-t),
$$

where $\mathrm{K}(t)$ and $\mathrm{K}^{\prime}(t)$ are the complete elliptic integrals of the first kind of parameters $t$ and $1-t$ respectively.

Now let us introduce Jacobi elliptic functions

$$
\operatorname{sn}(u, t)=\frac{1}{k^{1 / 2}} \frac{\theta_{1}(v \mid \tau)}{\theta_{4}(v \mid \tau)}, \quad \operatorname{cn}(u, t)=\frac{\left(k^{\prime}\right)^{1 / 2}}{k^{1 / 2}} \frac{\theta_{2}(v \mid \tau)}{\theta_{4}(v \mid \tau)}, \quad \operatorname{dn}(u, t)=\left(k^{\prime}\right)^{1 / 2} \frac{\theta_{3}(v \mid \tau)}{\theta_{4}(v \mid \tau)},
$$

where

$$
u=\frac{2 \mathrm{~K}(t)}{\pi} v
$$


and define the fundamental elliptic integral of the second kind [34] as

$$
\mathcal{E}(u, t)=\int_{0}^{u} \operatorname{dn}^{2}(x, t) d x
$$

It satisfies

$$
\mathcal{E}(\mathrm{K}(t), t)=\mathrm{E}(t)
$$

where $\mathrm{E}(t)$ is the complete elliptic integral of the second kind.

It is convenient to use slightly renormalized complete elliptic integrals

$$
K=\frac{2 \mathrm{~K}(t)}{\pi}, \quad E=\frac{2 \mathrm{E}(t)}{\pi} .
$$

One of the results of [33] can be written as

$$
\tau_{P}(t)=c_{0}(x, y) q^{y^{2} / \pi^{2}} t^{-1 / 4} \frac{\theta_{1}(x+\tau y \mid \tau)}{\theta_{4}(0 \mid \tau)}
$$

where $x$ and $y$ are two arbitrary parameters and $c_{0}(x, y)$ is a normalization constant independent of $t$.

First let us consider the high temperature regime $T>T_{c}$. Combining $(42,47,56)$ we obtain:

$$
C_{0}^{+}(t)=c_{0}(x, y) \frac{(1-t)^{1 / 4}}{t^{1 / 4}} q^{y^{2} / \pi^{2}} \frac{\theta_{1}(x+\tau y \mid \tau)}{\theta_{4}(0 \mid \tau)}
$$

for some values of $x$ and $y$. Recall that $C_{0}^{ \pm}(t)$ depends on the parameter $\lambda$. It remains to find a relationship between the parameters $x$ and $y$ on the RHS and the parameter $\lambda$ on the LHS of (57). We can expand both parts of (57) in series in $x, y$ and $\lambda$ and compare the expansions term by term.

Integrating the well known formula for a logarithmic derivative of $\theta_{4}(x \mid \tau)$ we obtain

$$
\theta_{4}(x \mid \tau)=\theta_{4}(0 \mid \tau) \exp \left\{-\frac{x^{2}}{2} K E+\int_{0}^{x K} \mathcal{E}(y, t) d y\right\} .
$$

Using (51) and (58) we can expand the rhs of (57) in a series in $x$ and $y \tau$ with polynomial coefficients depending on $t, K$ and $E$.

For $C_{0}^{+}(t)$ we need to calculate the first few form factors in the sum (7). To that end, we can use the integral representation (9) or a different integral representation [20] for the functions

$$
f_{N, N}^{(2 n+1)}=\hat{C}^{(2 n+1)}(N, N) / s, \quad f_{N, N}^{(2 n)}=\hat{C}^{(2 n)}(N, N) .
$$

It was observed in [20] that the diagonal form factors $f_{N, N}^{(n)}$ are polynomials in $t, K$ and $E$ for small values of $n$, and the first four, corresponding to $n=1 \ldots 4$ are given in [20]. Now we can expand the integral representation for the diagonal form-factors in series in $t$ up to sufficiently high order and compare it with the above polynomial Ansatz. In that way one can produce closed form expressions for the form-factors. The first few exact form factors $\hat{C}^{(n)}(0,0)$, $n=1,2, \ldots, 7$ are given in Appendix A. We note that in [4], eqn (5.7), Orrick et al. gave a conjectured expression for $\hat{C}^{(n)}(0,0)$ giving the results in terms of $q$ series. Evaluation of their expression for $n=1,2, \ldots, 7$ gives a series expansion that agrees with the expansion of the expressions given in Appendix A, thus providing mutual conformation. 
Now matching series on both sides of (57) up to order $\lambda^{8}$ we achieve perfect agreement if we choose

$$
c_{0}(x, y)=1, \quad \lambda=x-\frac{x^{3}}{6}+\frac{x^{5}}{120}-\frac{x^{7}}{5040}+O\left(x^{9}\right), \quad y=0
$$

From this expansion we can guess that

$$
\lambda=\sin (x)
$$

and we obtain

$$
C_{0}^{+}(t)=C^{+}(0,0 ; \lambda)=\frac{(1-t)^{1 / 4}}{t^{1 / 4}} \frac{\theta_{1}(\arcsin (\lambda) \mid \tau)}{\theta_{4}(0 \mid \tau)}
$$

a result which has not been published before. A nontrivial self-check of the relation (61) is to set $\lambda=1$ which corresponds to the symmetric Ising model.

We obtain

$$
C^{+}(0,0 ; 1)=\frac{(1-t)^{1 / 4}}{t^{1 / 4}} \frac{\theta_{1}\left(\frac{\pi}{2} \mid \tau\right)}{\theta_{4}(0 \mid \tau)}=\frac{(1-t)^{1 / 4}}{t^{1 / 4}} \frac{\theta_{2}(0 \mid \tau)}{\theta_{4}(0 \mid \tau)}=1
$$

as expected.

Another comment is in order. Based on connections to Painlevé VI theory we have proved that the generalized diagonal correlations can be represented in terms of theta-functions. However, the relations between the parameters $x, y$ and $\lambda$ are still conjectural. One of the possible ways to prove such relations is to find a partial differential equation for generalized correlations which includes derivatives with respect to both $\lambda$ and $t$.

We can handle the low-temperature regime $T<T_{c}$ in a similar way. The only difference is that now we have different relations between parameters,

$$
c_{0}(x, y)=-i \exp (i x), \quad \lambda=\sin (x), \quad y=\pi / 2 .
$$

The answer reads

$$
C_{0}^{-}(t)=C^{-}(0,0 ; \lambda)=(1-t)^{1 / 4} \frac{\theta_{4}(\arcsin (\lambda) \mid \tau)}{\theta_{4}(0 \mid \tau)}=\frac{\theta_{4}(\arcsin (\lambda) \mid \tau)}{\theta_{3}(0 \mid \tau)} .
$$

Again for $\lambda=1$

$$
C_{0}^{-}(t)=C^{-}(0,0 ; 1)=\frac{\theta_{4}\left(\frac{\pi}{2} \mid \tau\right)}{\theta_{3}(0 \mid \tau)}=1 .
$$

The formula (65) first appeared in Boukraa et al. [20] (see formula (92), Section 6). The authors of [20] remark that they have calculated $\lambda$-extended diagonal correlations for $N=$ $0,1,2, \ldots$, and have given a selection of the algebraic curves which arise from special choices of $\lambda{ }^{4}$

We emphasize that in our derivation of $(62,65)$ we relied on the exact calculation of taufunctions for the Picard solution [33]. This provides an alternative derivation of equation (65), previously announced in [20].

As mentioned above we have conjectured relations between parameters (60-61) and (64) based on series expansions in $\lambda$ and $x$ and the Ising case $\lambda=1$. However, formulae (63) and (65) produce analytic expressions for the integrals (9) at $M=N=0$ for any positive integer $n$. We checked numerically up to $n=10$ that we obtain correct values for the form factors in (9).

\footnotetext{
${ }^{4}$ After this paper appeared, results for $N=0,1$ diagonal correlations were given in [23], which follow from conjectured results in [4].
} 
Now we turn to the calculation of $C_{1}^{ \pm}(t)$. Let us start with the auxiliary Hamiltonian $h_{0}(t)$ corresponding to the Picard solution. We take the tau-function (56) with $y=0(y=\pi / 2)$ and arbitrary $x$ for $T>T_{c}\left(T<T_{c}\right)$ and use eqns. (20-21) with parameters (40) to find $h_{0}(t)$. Now we can find auxiliary Hamiltonians $h_{ \pm}(t)$ corresponding to $N= \pm 1$ from (31-32). It is easy to see that they coincide

$$
h_{+}(t)=h_{-}(t) .
$$

It follows from (20-21) and (37) with $N= \pm 1$ that

$$
\tau_{1}(t)=a(x) t \tau_{-1}(t)
$$

and from (42)

$$
C_{1}^{ \pm}(t)=a_{ \pm}(x) C_{-1}^{ \pm}(t)
$$

for some integration constants $a_{ \pm}(x)$.

Substituting into (43) at $N=0$ we obtain

$$
\left(C_{1}^{ \pm}(t)\right)^{2}=-\frac{4(1-t)^{2}}{a_{ \pm}(x)}\left(C_{0}^{ \pm}(t)\right)^{2}\left\{t \frac{d^{2}}{d t^{2}} \log C_{0}^{ \pm}(t)+\frac{d}{d t} \log C_{0}^{ \pm}(t)\right\} .
$$

We can calculate first and second derivatives of $C_{0}^{ \pm}(t)$. For that we need the following differentiation formulae

$$
\begin{gathered}
\frac{d}{d t} K=\frac{E}{2 t(1-t)}-\frac{K}{2 t}, \quad \frac{d}{d t} E=\frac{E-K}{2 t}, \\
\frac{d}{d t} \operatorname{sn}(u, t)=-\frac{\operatorname{sn}(u, t) \operatorname{cn}^{2}(u, t)}{2(t-1)}+\frac{\operatorname{cn}(u, t) \operatorname{dn}(u, t)}{2 t(t-1)}[u(t-1)+\mathcal{E}(u, t)], \\
\frac{d}{d t} \operatorname{cn}(u, t)=\frac{\operatorname{sn}^{2}(u, t) \operatorname{cn}(u, t)}{2(t-1)}-\frac{\operatorname{sn}(u, t) \operatorname{dn}(u, t)}{2 t(t-1)}[u(t-1)+\mathcal{E}(u, t)], \\
\frac{d}{d t} \operatorname{dn}(u, t)=\frac{\operatorname{sn}^{2}(u, t) \operatorname{dn}(u, t)}{2(t-1)}-\frac{\operatorname{sn}(u, t) \operatorname{cn}(u, t)}{2(t-1)}[u(t-1)+\mathcal{E}(u, t)], \\
\frac{d}{d t} \mathcal{E}(u, t)=\frac{\mathcal{E}^{2}(u, t)}{2(t-1)}-\frac{u}{2} \operatorname{sn}^{2}(u, t)-\frac{\operatorname{sn}(u, t) \operatorname{cn}(u, t) \operatorname{dn}(u, t)}{2(t-1)}
\end{gathered}
$$

and

$$
\frac{d}{d t}\left[\log \left(\frac{\theta_{4}(x \mid \tau)}{\theta_{4}(0 \mid \tau)}\right)\right]=\frac{\operatorname{sn}^{2}(x K, t)}{4(1-t)}+\frac{(\mathcal{E}(x K, t)-x E)^{2}}{4 t(t-1)} .
$$

The last formula can be proved using (58) and a partial differential equation for theta-functions.

After straightforward simplifications we arrive at

$$
t \frac{d^{2}}{d t^{2}} \log C_{0}^{-}(t)+\frac{d}{d t} \log C_{0}^{-}(t)=-\frac{1}{4(1-t)^{2}}(\operatorname{cn}(z, t) \operatorname{dn}(z, t)+\operatorname{sn}(z, t) X)^{2}
$$

and

$$
t \frac{d^{2}}{d t^{2}} \log C_{0}^{+}(t)+\frac{d}{d t} \log C_{0}^{+}(t)=-\frac{1}{4 t(1-t)^{2}} \frac{X^{2}}{\operatorname{sn}^{2}(z, t)}
$$

where

$$
z=x K=\frac{2 x \mathrm{~K}(t)}{\pi}, \quad X=\frac{1}{K}\left[\log \theta_{4}(x \mid \tau)\right]_{x}^{\prime}=\mathcal{E}(x K, t)-x E
$$


Substituting back into (70) we have

$$
C_{1}^{-}(t)=\frac{1}{\sqrt{a_{-}(x)}} \frac{\theta_{4}(x \mid \tau)}{\theta_{3}(0 \mid \tau)}(\operatorname{cn}(z, t) \operatorname{dn}(z, t)+\operatorname{sn}(z, t) X)
$$

and

$$
C_{1}^{+}(t)=\frac{1}{\sqrt{a_{+}(x)}} \frac{\theta_{4}(x \mid \tau)}{\theta_{3}(0 \mid \tau)} \frac{X}{t^{1 / 2}} .
$$

To determine the factors $a_{ \pm}(x)$ we use (61), expand (80-81) in $\lambda$ and compare with form factor expansions (7-8) at $M=N=1$. These form-factors are given in [20] up to order nine in $\lambda$ and we will not reproduce them here. All these expansions are consistent with a choice

$$
\sqrt{a_{+}(x)}=\sqrt{a_{-}(x)}=\cos (x) .
$$

Again we can do an independent check by expanding near the point $x=\pi / 2$ or $\lambda=1$ which corresponds to the symmetric Ising model. In this case we obtain the results for the nearest-neighbor diagonal correlations $C(1,1)$

$$
C^{+}(1,1 ; 1)=t^{-1 / 2}[(t-1) K+E]
$$

and

$$
C^{-}(1,1 ; 1)=E,
$$

which agree with formulae (4.9a) and (4.9b), Section VIII.4 of [11].

Now we can substitute the initial values of $C_{0}^{ \pm}(t), C_{1}^{ \pm}(t)$ into recurrence relation (43) and calculate $C_{N}^{ \pm}(t)$ for any $N=2,3, \ldots$ for arbitrary $\lambda$. All we need are the differentiation formulae given above.

We obtain for $N=2$ and $T<T_{c}$

$$
C_{2}^{-}(t)=\frac{\theta_{4}(x \mid \tau)}{\cos ^{2}(x) \theta_{3}(0 \mid \tau)}\left[C^{2}+\frac{8}{3} C S X+X^{2}\left(2 S^{2}-\frac{t+1}{3 t}\right)-\frac{X^{4}}{3 t}\right]
$$

and for $T>T_{c}$

$$
\begin{aligned}
C_{2}^{+}(t) & =-\frac{\theta_{4}(x \mid \tau)}{3 t \cos ^{2}(x) \theta_{3}(0 \mid \tau)}\left[2 C X\left[2 X^{2}+2 t S^{2}-t-1\right]+\right. \\
& +S\left[X^{4}+X^{2}\left(6 t S^{2}-5(t+1)\right]+t C^{2}\right]
\end{aligned}
$$

where $X$ is defined in (79), and

$$
C=\operatorname{cn}(z, t) \operatorname{dn}(z, t), \quad S=\operatorname{sn}(z, t) .
$$

The answer for $C_{3}^{ \pm}(t)$ is given in Appendix B.

Let us point out that the factor $C_{N-1}^{ \pm}(t)$ always divides the lhs of (43). We don't have a rigorous proof of this cancelation. Exactly the same phenomenon occurs for many polynomial tau-functions related to Painlevé equations. Usually the proof of this fact relies on determinant representations of such tau-functions. It would be extremely interesting to find a determinant representation of $C_{N}^{ \pm}(t)$ for arbitrary $\lambda$.

As mentioned in the Introduction, the equations (43) for $\lambda=1$ together with initial conditions $(63,66,83-84)$ and differentiation formulae (71) give a very efficient procedure for the calculation of diagonal correlation functions $C^{ \pm}(N, N)$ for the symmetric Ising model. 


\section{A calculation of $C^{ \pm}(0,1 ; \lambda)$}

In this section we perform a resummation of the $\lambda$-extension of the simplest row nearestneighbor correlation $C^{ \pm}(0,1 ; \lambda)$. It is not known whether off-diagonal correlations and their $\lambda$-extensions satisfy nonlinear differential equations. Therefore, a knowledge of explicit expressions for $C^{ \pm}(M, N ; \lambda)$ at small values of $M, N$ can be useful.

First we shall start with the expansion of integrals (9) at $s \rightarrow 0$. We have

$$
\begin{aligned}
& \sinh \gamma_{i}=s^{-1}\left(1-s \cos \omega_{i}+O\left(s^{2}\right)\right), \quad x_{i}=\frac{s}{2}\left(1+s \cos \omega_{i}+O\left(s^{2}\right)\right), \\
& h_{i j}^{2}=\sin ^{2}\left(\frac{\omega_{i}-\omega_{j}}{2}\right) s^{2}\left(1+s\left(\cos \omega_{i}+\cos \omega_{j}\right)+O\left(s^{2}\right)\right) .
\end{aligned}
$$

Then it is easy to see that $\hat{C}^{(n)}(0,1)$ has a kinematic zero of order $n^{2}$ as $s \rightarrow 0$.

Expanding $\hat{C}^{(n)}(0,1)$ in $s$ for $n=1,2,3,4,5,6$ one can see that $\hat{C}^{(n)}(0,1)$ has a zero in $s$ of order $n(n+1)$ and guess the first two nonzero terms of the expansion

$$
\hat{C}^{(n)}(0,1)=\frac{s^{n(n+1)}}{2^{n^{2}}}\left(1+\frac{s^{2}}{4}+O\left(s^{4}\right)\right) \quad \text { at } \quad s \rightarrow 0, \quad n=1,2,3, \ldots
$$

We expect these integrals to be polynomials in $K, E$ and $s^{2}$ [20]. The case $n=1$ is easy

$$
\hat{C}^{(1)}(0,1)=-\frac{1}{2}+\frac{1}{2}\left(1+s^{2}\right) K
$$

and the answer for $n=2$ was given in [20]

$$
\hat{C}^{(2)}(0,1)=\frac{3}{8}-\frac{1}{4}\left(1+s^{2}\right) K-\frac{1}{8}\left(s^{2}-3\right)\left(1+s^{2}\right) K^{2}-\frac{1}{2} E K .
$$

Using series expansions in $s$ up to a sufficiently high order we were able to obtain similar expressions for $n=3,4$. They are given in Appendix B. The structure of the $n=1,2,3,4$ cases suggests the following Ansatz for general $n$ :

$$
\hat{C}^{(n)}(0,1)=\sum_{i=0}^{\left[\frac{n}{2}\right]} a_{i}^{(n)}(E K)^{i}+\left(1+s^{2}\right) \sum_{i=1}^{n} \sum_{j=0}^{\left[\frac{i-1}{2}\right]} b_{i, j}^{(n)} E^{j} K^{i-j} p_{i-2 j}(s)
$$

where $[x]$ stands for the integer part of $x, a_{i}^{(n)}$ and $b_{i, j}^{(n)}$ are rational coefficients, and $p_{n}(s)$ is the polynomial in $s^{2}$ of degree $n-1$ with integer coefficients:

$$
p_{n}(s)=c_{0, n}+c_{1, n} s^{2}+\ldots+c_{n-2, n} s^{2(n-2)}+s^{2(n-1)} .
$$

It is quite remarkable that the condition for the sum in (92) to have a zero of order $n(n+1)$ in $s$ fixes all coefficients in (92) together with polynomials $p_{n}(s)$ up to a common normalization factor. This factor is determined by the coefficient of the leading term in (89).

Applying this procedure we easily calculated $\hat{C}^{(n)}(0,1)$ for $n=5,6, \ldots, 15$. The coefficients $a_{i}^{(n)}$ and $b_{i, j}^{(n)}$ together with polynomials $p_{n}(s)$ up to $n=6$ are listed in Appendix B.

Of course, this is just experimental mathematics based on matching series expansions. It is desirable to run some self-consistency tests. We evaluated the integrals $\hat{C}^{(n)}(0,1)$ for $n=1, \ldots, 6$ numerically at $s=0.95$ and they matched (92) with 1 part in $10^{-8}$ accuracy which corresponds to approximately 360 terms in the series expansions in $s$. So the Ansatz (92) is almost certainly 
correct. Note that eqn (5.8) in Orrick et al. [4] gives a conjectured expression for $\hat{C}^{(n)}(0,1)$ in terms of Jacobi theta functions and $q$ series. Expanding that equation in a $q$-series and converting the series expansions of (B1) to (B7) to $q$-series provides a valuable consistency check. We confirm that the series agree to the first 100 terms.

Now having explicit expressions for the form-factors we can try to perform a resummation of the next-neighbor correlations $C(0,1 ; \lambda)$.

As we did for the diagonal correlations, it is natural to look at the the logarithms of $C^{ \pm}(0,1 ; \lambda)$ and expand them in series in $x$, where

$$
x=\arcsin (\lambda) .
$$

Then we obtain

$$
\log C^{ \pm}(0,1 ; x)=-\frac{K E}{2} x^{2}+f^{ \pm}\left(x, K, s^{2}\right)
$$

where functions $f^{ \pm}\left(x, K, s^{2}\right)$ as series in $x$ do not depend on the elliptic integral of the second kind $E$. The absence of $E$ in the functions $f^{ \pm}\left(x, K, s^{2}\right)$ is a very strong argument in favour of our Ansatz (92).

Using definitions (7-8) and (58) we can write

$$
C^{+}(0,1 ; x)=\frac{1}{s} \frac{\theta_{4}(x \mid \tau)}{\theta_{3}(0 \mid \tau)} F^{+}\left(x, K, s^{2}\right), \quad C^{-}(0,1 ; x)=\frac{\theta_{4}(x \mid \tau)}{\theta_{3}(0 \mid \tau)} F^{-}\left(x, K, s^{2}\right) .
$$

Now let us rewrite series expansions for $F^{ \pm}\left(x, K, s^{2}\right)$ in variables $x$ and $y=x K$ and split them into even and odd parts in $y$. It appears that the resulting two functions factorize and have similar structure

$$
F^{ \pm}\left(x, K, s^{2}\right)=\alpha_{ \pm}(x)\left[g_{\text {even }}\left(x K, s^{2}\right)-\beta_{ \pm}(x) g_{\text {odd }}\left(x K, s^{2}\right)\right] .
$$

where $\alpha_{ \pm}(x)$ and $\beta_{ \pm}(x)$ depend on the variable $x$ only and $g_{\text {even }}\left(y, s^{2}\right), g_{o d d}\left(y, s^{2}\right)$ do not depend on $x$. A similar separation happens for generalized diagonal correlations at $N=0,1$ and is another strong argument in favour of our calculations.

Using Ansatz (92) we calculated form factors (9) for $n=1, \ldots, 15$ and obtained series expansions for functions $\alpha_{ \pm}(x)$ and $\beta_{ \pm}(x)$ up to $x^{15}$. Then it is easy to guess that

$$
\alpha_{+}(x)=-\frac{\sin \left(\frac{x}{2}\right)}{\cos (x)}, \quad \alpha_{-}(x)=\frac{\cos \left(\frac{x}{2}\right)}{\cos (x)}, \quad \beta_{-}(x)=\frac{1}{\beta_{+}(x)}=\tan \left(\frac{x}{2}\right) .
$$

Finally we have to find the functions $g_{\text {even }}\left(y, s^{2}\right)$ and $g_{\text {odd }}\left(y, s^{2}\right)$ where the variable $y=x K$. Their expansions in $y$ have polynomial coefficients in $s^{2}$ of growing complexity and it is not easy to reconstruct them.

However, based on the diagonal case we assumed that these functions are double-periodic in $y$ and substituted their expansions into the differential equation

$$
\left(\partial_{y} g(y, s)\right)^{2}=\sum_{i=0}^{4} A_{i}(s)(g(y, s))^{i},
$$

where the coefficients $A_{i}(s)$ are independent of the variable $y$.

This leads to immediate success, and we can easily find the polynomial coefficients $A_{i}(s)$ and solve the differential equation (99) for both cases. 
Collecting all the above formulae we get the final answer

$$
\begin{aligned}
& C^{-}(0,1 ; x)=\frac{\theta_{4}(x \mid q)}{\theta_{3}(0 \mid q)} \frac{\cos \left(\frac{x}{2}\right)}{\cos (x)}\left[g_{e v}(x, s)-\tan \left(\frac{x}{2}\right) g_{\text {odd }}(x, s)\right], \\
& C^{+}(0,1 ; x)=-\frac{1}{s} \frac{\theta_{4}(x \mid q)}{\theta_{3}(0 \mid q)} \frac{\sin \left(\frac{x}{2}\right)}{\cos (x)}\left[g_{e v}(x, s)-\cot \left(\frac{x}{2}\right) g_{\text {odd }}(x, s)\right],
\end{aligned}
$$

where

$$
g_{e v}(x, s)=\frac{\operatorname{cn}\left(\frac{x K}{2}, s^{4}\right) \operatorname{dn}\left(\frac{x K}{2}, s^{4}\right)}{1-s^{2} \operatorname{sn}\left(\frac{x K}{2}, s^{4}\right)^{2}}, \quad g_{\text {odd }}(x, s)=\frac{\left(1+s^{2}\right) \operatorname{sn}\left(\frac{x K}{2}, s^{4}\right)}{1+s^{2} \operatorname{sn}\left(\frac{x K}{2}, s^{4}\right)^{2}}
$$

and the variable $x$ is related to $\lambda$ by (61) or (94).

The final check is to expand (100) near $x=\pi / 2$ which corresponds to the symmetric Ising model and compare the answer with known nearest-neighbor row pair correlation functions. We obtain

$$
C^{+}(0,1)=\frac{\sqrt{1+s^{2}}}{2 s}\left(1+\left(s^{2}-1\right) K\right)
$$

and

$$
C^{-}(0,1)=\frac{\sqrt{1+s^{2}}}{2}\left(1-\left(s^{2}-1\right) K\right)
$$

which match (4.5a) and (4.5b) of Section VIII.4, [11].

Let us briefly comment on the formulae (100). To our knowledge closed expressions for generalized diagonal correlations $C^{ \pm}(0,1)$ have not appeared in the literature before.

As discussed in in the introduction in $1980 \mathrm{McCoy}$, Perk and Wu obtained quadratic difference relations for correlation functions. In our notation the $\lambda$-extension of these equations is given in [4]. These difference equations allow one to calculate generalized correlation functions provided that we know the answer for diagonal correlations and the first off-diagonal pair correlations $C(0,1)$. Therefore, formulae (100) provide the initial conditions for a recursive calculation of all generalized pair correlation functions $C(M, N ; \lambda)$ for arbitrary $M, N=0,1,2, \ldots$

While undoubtedly correct, a rigorous proof is still lacking. The missing ingredient is, for example, a Toda type relation similar to that obtained above for the diagonal correlations, plus verification of the connection between the parameters $\lambda$ and $x$. This is currently under investigation.

After the appearance of the first draft of this paper, it was pointed out to us by W.P. Orrick and J-M. Maillard that the result we give in eqn (100) is, in principle, derivable from eqn (5.8) in the paper [4] by Orrick et al. by summing over the correlation functions and making appropriate substitutions. We have not confirmed this result. We remark however that if that is indeed the case, further exact expressions for $C(1,1 ; \lambda), C(2,0 ; \lambda)$, and $C(2,1 ; \lambda)$ should be similarly derivable from eqns (5.9)-(5.11) of [4] and consistent with Perk's difference equations.

\section{Conclusions and outlook}

In this paper we have found and proved a set of Toda-type difference equations (6) satisfied by diagonal pair correlation functions. It is interesting that the $\lambda$ - extension of these pair correlations satisfy the same equations. We think that there should be a direct way to prove relations (6) from the Toeplitz determinant representation of the diagonal correlations. That would then enable us to understand the determinantal representation for their $\lambda$-extension.

When that is done, we could try to generalize relations (6) for the case of row correlations where the Toeplitz determinant representation is known for $\lambda=1$. However, it could well be 
difficult to generalize it to arbitrary values of $\lambda$. In fact, formulae (100) provide the first examples of closed form expressions for the $\lambda$ - extended nearest-neighbor row correlation functions. We failed to find a nonlinear differential equation satisfied by (100) valid for any value of $\lambda$. Of course, that doesn't mean that such an equation doesn't exist.

Let us emphasize that based on connections to Painlevé VI theory we have proved the results in equations $(57,65)$, up to the non-rigorous identification of the connection between the parameter $\lambda$ and the parameters $x$ and $y$.

We also note that in 2005 Forrester and Witte [35] gave a nonlinear system of recurrences in their corollary (6.3), related to the discrete Painlevé V equation for the same ratio of correlations that appears on the rhs of (43).

Finally, as we mentioned above, the derivation of the generalized diagonal correlations and relations (100) is not rigorous, based as it is on series expansions. However it is undoubtedly correct, satisfying numerous checks, and should provide initial conditions to calculate all generalized pair correlations $C(M, N ; \lambda)$ for any $M$ and $N$ from the quadratic difference equations of Perk. This should provide all necessary tools for a significantly more efficient generation of the series expansion for the $\lambda$-extended susceptibility. An ambitious, but still distant hope is to sum the correlation function series and produce the long sought closed-form expression for the susceptibility. We are planning to report on the evaluation of $C(M, N ; \lambda)$ in a forthcoming publication.

\section{Acknowledgments}

The authors thank V.V. Bazhanov, M.T. Batchelor, J. de Gier and S. Sergeev for valuable comments and interesting discussions while this work was being conducted, and P. J. Forrester, B.G Nickel, J-M Maillard, B M Mc Coy, B.G Nickel and W P Orrick for valuable comments

which significantly improved the manuscript. This work is supported by the Australian Research Council.

\section{Appendix A. The diagonal case}

In this Appendix we present the first seven form factors (43) at $M=N=0$ used for the calculation of initial conditions for the recursion relations (6). We also give generalized diagonal correlation functions for $N=3$. 
Form factors $\hat{C}^{(n)}(0,0)$ for $n=1, \ldots, 7$ :

$$
\begin{aligned}
& \frac{\hat{C}^{(1)}(0,0)}{s K}=1 \\
& \frac{\hat{C}^{(2)}(0,0)}{K}=\frac{K}{2}-\frac{E}{2}, \\
& \frac{\hat{C}^{(3)}(0,0)}{s K}=\frac{1}{6}-\frac{1}{2} K E-\frac{1}{6}(t-2) K^{2}, \\
& \frac{\hat{C}^{(4)}(0,0)}{K}=\frac{K}{6}+\frac{K^{3}}{8}-\frac{t K^{3}}{12}-\frac{E}{6}-\frac{K^{2} E}{4}+\frac{K E^{2}}{8} \\
& \frac{\hat{C}^{(5)}(0,0)}{s K}=\frac{3}{40}-\frac{K E}{4}+\frac{K^{2} E^{2}}{8}-\frac{1}{12}(t-2) K^{2}(1-K E)+\frac{1}{120}\left(t^{2}-6 t+6\right) K^{4} \\
& \frac{\hat{C}^{(6)}(0,0)}{K}=\frac{4 K}{45}+\frac{K^{3}}{12}\left(1-\frac{2 t}{3}\right)+\frac{K^{5}}{48}\left(1-\frac{22 t}{15}+\frac{8 t^{2}}{15}\right)+ \\
& +E\left(-\frac{4}{45}+\frac{K E}{12}-\frac{K^{2} E^{2}}{48}-\frac{K^{2}}{6}+\frac{K^{3} E}{16}+\frac{t K^{4}}{24}-\frac{K^{4}}{16}\right) \\
& \frac{\hat{C}^{(7)}(0,0)}{s K}=\frac{5}{112}-\frac{37 K E}{240}+\frac{5 K^{2} E^{2}}{48}-\frac{K^{3} E^{3}}{48}-(t-2) K^{2}\left(\frac{37}{720}-\frac{5 K E}{72}+\frac{K^{2} E^{2}}{48}\right)+ \\
& +\left(t^{2}-6 t+6\right) K^{4}\left(\frac{1}{144}-\frac{K E}{240}\right)-\frac{1}{5040}(t-2)\left(t^{2}-10 t+10\right) K^{6}
\end{aligned}
$$

In [36] it is pointed out by Bostan et al. that the minimal order ODE for the $n$-particle contributions to the susceptibility $\tilde{\chi}^{(n)}$ are achieved by appropriate linear combinations of $\tilde{\chi}^{(n)}$ with terms $\tilde{\chi}^{(n-2)}, \tilde{\chi}^{(n-4)}$, etc. In particular, these combinations are: $\tilde{\chi}^{(3)}-\tilde{\chi}^{(1)} / 6, \tilde{\chi}^{(4)}-\tilde{\chi}^{(2)} / 3$, $\tilde{\chi}^{(5)}-\tilde{\chi}^{(3)} / 2+\tilde{\chi}^{(1)} / 120, \tilde{\chi}^{(6)}-2 \tilde{\chi}^{(4)} / 3+\tilde{\chi}^{(2)} / 45$. It is remarkable that the analogous combinations of form factors $\hat{C}^{(n)}(0,0)$ for $n=1, \ldots, 7$ give expressions that are homogeneous in products of elliptic integrals of degree $n .{ }^{5}$ That is to say, combinations such as $\hat{C}^{(6)}(0,0)-2 \hat{C}^{(4)}(0,0) / 3+$ $\hat{C}^{(2)}(0,0) / 45$ contain only terms of the form $K^{6}, K^{5} E, K^{4} E^{2}$ and $K^{3} E^{3}$. From (A.2) it is easy to conjecture that the appropriate linear combination that will give the minimum order ODE for $\tilde{\chi}^{(7)}$ is $\tilde{\chi}^{(7)}-5 \tilde{\chi}^{(5)} / 3 !+13 \tilde{\chi}^{(3)} / 5 !-\tilde{\chi}^{(1)} / 7$ !

Generalized diagonal correlations $C_{3}^{ \pm}(t)$ are obtained by solving (6) for $N=2$. They are:

$$
C_{3}^{+}(t)=\frac{\theta_{4}(x \mid \tau)}{135 t^{5 / 2} \cos ^{3}(x) \theta_{3}(0 \mid \tau)}\left[t C S R_{1}^{+}+X R_{2}^{+}\right]
$$

and

$$
C_{3}^{-}(t)=-\frac{\theta_{4}(x \mid \tau)}{135 t^{2} \cos ^{3}(x) \theta_{3}(0 \mid \tau)}\left[C R_{1}^{-}+S X R_{2}^{-}\right]
$$

\footnotetext{
${ }^{5}$ This was pointed out to us by B G Nickel.
} 


$$
\begin{aligned}
& R_{1}^{+}(t)=168 X^{6}+84 X^{4}\left[6 t S^{2}-7(t+1)\right]+4 t\left(1-S^{2}\right)\left(1-t S^{2}\right)\left(2 t S^{2}-9(t+1)\right) \\
& +108 X^{2}\left[2 t S^{2}\left(t S^{2}-3(t+1)\right)+3+4 t+3 t^{2}\right] \\
& R_{2}^{+}(t)=-X^{8}+6 X^{6}\left[6 t S^{2}-t-1\right]+9 X^{4}\left[42 t S^{2}\left(t S^{2}-t-1\right)-t^{2}+28 t-1\right] \\
& +2 X^{2}\left[105 t^{2} S^{4}\left(2 t S^{2}-5(t+1)\right)+3 t S^{2}\left(99+196 t+99 t^{2}\right)-(t+1)\left(2+103 t+2 t^{2}\right)\right] \\
& +3 t\left(1-S^{2}\right)\left(1-t S^{2}\right)\left[7 t S^{2}\left(3 t S^{2}-11(t+1)\right)+24 t^{2}+21 t+24\right] \\
& R_{1}^{-}(t)=9 X^{8}+42 X^{6}\left[2 t S^{2}-t-1\right]+3 X^{4}\left[14 t S^{2}\left(3 t S^{2}-t-1\right)-13 t^{2}+28 t-13\right] \\
& +6 X^{2}\left[3 t^{2} S^{4}\left(2 t S^{2}+t+1\right)-3 t S^{2}\left(3+52 t+3 t^{2}\right)+(t+1)\left(2+13 t+2 t^{2}\right)\right] \\
& +t^{2}\left(1-S^{2}\right)\left(1-t S^{2}\right)\left[t S^{4}+3 S^{2}(t+1)-135\right], \\
& R_{2}^{-}(t)=X^{8}+6 X^{6}\left[6 t S^{2}-5(t+1)\right]+9 X^{4}\left[14 t S^{2}\left(t S^{2}-t-1\right)+t^{2}+28 t+1\right] \\
& +2 X^{2}\left[21 t^{2} S^{4}\left(2 S^{2} t-t-1\right)-3 t S^{2}\left(13+84 t+13 t^{2}\right)+(t+1)\left(20+61 t+20 t^{2}\right)\right] \\
& +3 t\left(1-S^{2}\right)\left(1-t S^{2}\right)\left[t S^{2}\left(3 t S^{2}+5(t+1)\right)-4 t^{2}-197 t-4\right] .
\end{aligned}
$$

\section{Appendix B. The off-diagonal case}

In this Appendix we first give form factors $\hat{C}^{(n)}(0,1)$ for $n=1,2,3,4$ obtained by series expansions of (9) to series in $s$ up to 200 terms.

$$
\begin{aligned}
\hat{C}^{(1)}(0,1)= & -\frac{1}{2}+\frac{1}{2}\left(1+s^{2}\right) K \\
\hat{C}^{(2)}(0,1)= & \frac{3}{8}-\frac{1}{4}\left(1+s^{2}\right) K-\frac{1}{8}\left(s^{2}-3\right)\left(1+s^{2}\right) K^{2}-\frac{1}{2} E K \\
\hat{C}^{(3)}(0,1)= & -\frac{5}{16}+\frac{13}{48}\left(1+s^{2}\right) K+\frac{1}{16}\left(s^{2}-3\right)\left(1+s^{2}\right) K^{2}- \\
& -\frac{1}{48}\left(s^{4}+6 s^{2}-11\right)\left(1+s^{2}\right) K^{3}+\frac{1}{4} E K-\frac{1}{4} E K^{2}\left(1+s^{2}\right) \\
\hat{C}^{(4)}(0,1)= & \frac{35}{128}-\frac{19}{96}\left(1+s^{2}\right) K-\frac{17}{192}\left(s^{2}-3\right)\left(1+s^{2}\right) K^{2}+ \\
& +\frac{1}{96}\left(s^{4}+6 s^{2}-11\right)\left(1+s^{2}\right) K^{3}+\frac{1}{384}\left(s^{6}-21 s^{4}+3 s^{2}+25\right)\left(1+s^{2}\right) K^{4}
\end{aligned}
$$

We note that, in analogy to the linear combinations of form factors $\hat{C}^{(n)}(0,0)$ for $n=1, \ldots, 7$ that give expressions that are homogeneous in products of elliptic integrals of degree $n$ reported in Appendix A, rather more complicated combinations of form factors $\hat{C}^{(n)}(0,1)$ produce similar results for the form factors $\hat{C}^{(n)}(0,1)$. In particular, we note that

$$
\hat{C}^{(3)}(0,1)+\frac{1}{2} \hat{C}^{(2)}(0,1)-\frac{7}{24} \hat{C}^{(1)}(0,1)-\frac{1}{48}=\mathrm{O}\left(E K^{2}, K^{3}\right)
$$




\begin{tabular}{|c|c|c|c|c|}
\hline$n$ & $a_{0}^{(n)}$ & $a_{1}^{(n)}$ & $a_{2}^{(n)}$ & $a_{3}^{(n)}$ \\
\hline 6 & $\frac{231}{1024}$ & $-\frac{3319}{11520}$ & $\frac{25}{192}$ & $-\frac{1}{48}$ \\
\hline 5 & $-\frac{63}{256}$ & $\frac{23}{96}$ & $-\frac{1}{16}$ & \\
\hline 4 & $\frac{35}{128}$ & $-\frac{17}{48}$ & $\frac{1}{8}$ & \\
\hline 3 & $-\frac{5}{16}$ & $\frac{1}{4}$ & & \\
\hline 2 & $\frac{3}{8}$ & $-\frac{1}{2}$ & & \\
\hline 1 & $-\frac{1}{2}$ & & & \\
\hline
\end{tabular}

Table 1: Coefficients $a_{i}^{(n)}$ for $n=1, \ldots, 6$.

\begin{tabular}{|c|c|c|c|c|c|c|c|c|c|c|c|c|}
\hline$n$ & $b_{1,0}^{(n)}$ & $b_{2,0}^{(n)}$ & $b_{3,0}^{(n)}$ & $b_{3,1}^{(n)}$ & $b_{4,0}^{(n)}$ & $b_{4,1}^{(n)}$ & $b_{5,0}^{(n)}$ & $b_{5,1}^{(n)}$ & $b_{5,2}^{(n)}$ & $b_{6,0}^{(n)}$ & $b_{6,1}^{(n)}$ & $b_{6,2}^{(n)}$ \\
\hline 6 & $-\frac{1289}{7680}$ & $-\frac{3319}{46080}$ & $\frac{3}{256}$ & $\frac{9}{64}$ & $\frac{25}{9216}$ & $\frac{25}{384}$ & $-\frac{1}{7680}$ & $-\frac{1}{192}$ & $-\frac{1}{32}$ & $-\frac{1}{46080}$ & $-\frac{1}{768}$ & $-\frac{1}{64}$ \\
\hline 5 & $\frac{263}{1280}$ & $\frac{23}{384}$ & $-\frac{7}{384}$ & $-\frac{7}{32}$ & $-\frac{1}{768}$ & $-\frac{1}{32}$ & $\frac{1}{3840}$ & $\frac{1}{96}$ & $\frac{1}{16}$ & & & \\
\hline 4 & $-\frac{19}{96}$ & $-\frac{17}{192}$ & $\frac{1}{96}$ & $\frac{1}{8}$ & $\frac{1}{384}$ & $\frac{1}{16}$ & & & & & & \\
\hline 3 & $\frac{13}{48}$ & $\frac{1}{16}$ & $-\frac{1}{48}$ & $-\frac{1}{4}$ & & & & & & & & \\
\hline 2 & $-\frac{1}{4}$ & $-\frac{1}{8}$ & & & & & & & & & & \\
\hline 1 & $\frac{1}{2}$ & & & & & & & & & & & \\
\hline
\end{tabular}

Table 2: Coefficients $b_{i, j}^{(n)}$ for $n=1, \ldots, 6$

and

$$
\hat{C}^{(4)}(0,1)+\frac{1}{2} \hat{C}^{(3)}(0,1)-\frac{11}{24} \hat{C}^{(2)}(0,1)-\frac{5}{48} \hat{C}^{(1)}(0,1)+\frac{1}{384}=\mathrm{O}\left(E K^{3}, E^{2} K^{2}, K^{4}\right) .
$$

Here we also list coefficients $a_{i}^{(n)}, b_{i, j}^{(n)}$ and polynomials $p_{n}(s)$ for $n=1, \ldots, 6$ which occur in eqn (92) for the form factors $\hat{C}^{(n)}(0,1)$.

$$
\begin{aligned}
& p_{1}(s)=1 \\
& p_{2}(s)=-3+s^{2} \\
& p_{3}(s)=-11+6 s^{2}+s^{4} \\
& p_{4}(s)=25+3 s^{2}-21 s^{4}+s^{6} \\
& p_{5}(s)=201-180 s^{2}-66 s^{4}+60 s^{6}+s^{8} \\
& p_{6}(s)=-299-123 s^{2}+466 s^{4}+106 s^{6}-183 s^{8}+s^{10}
\end{aligned}
$$




\section{References}

[1] Onsager, L. Crystal statistics. I. A two-dimensional model with an order-disorder transition. Phys. Rev. (2) 65 (1944) 117-149.

[2] Onsager, L. Discussion remark (Spontaneous magnetisation of the two-dimensional Ising model). Nuovo Cimento (Suppl.) 6 (1949) 261.

[3] Yang, C. N. The spontaneous magnetization of a two-dimensional Ising model. Physical Rev. (2) 85 (1952) 808-816.

[4] Orrick, W. P., Nickel, B., Guttmann, A. J., and Perk, J. H. H. The susceptibility of the square lattice Ising model: new developments. In Proceedings of the Baxter Revolution in Mathematical Physics (Canberra, 2000), J. Statist. Phys., volume 102, pages 795-841, 2001.

[5] McCoy, B. M. and Wu, T. T. Nonlinear partial difference equations for the two-dimensional Ising model. Phys. Rev. Lett. 45 (1980) 675-678.

[6] McCoy, B. M., Perk, J. H. H., and Wu, T. T. Ising field theory: quadratic difference equations for the $n$-point Green's functions on the lattice. Phys. Rev. Lett. 46 (1981) $757-760$.

[7] Perk, J. H. H. Quadratic identities for Ising model correlations. Phys. Lett. A 79 (1980) $3-5$.

[8] Wu, T. T., McCoy, B. M., Tracy, C. A., and Barough, E. Spin-spin correlation functions for the two-dimensional Ising model: Exact theory in the scaling region. Phys. Rev. B 13 (1976) 316-374.

[9] Kaufman, B. and Onsager, L. Crystal Statistics. III. Short-Range Order in a Binary Ising Lattice. Phys. Rev. 76 (1949) 1244-1252.

[10] Montroll, E. W., Potts, R. B., and Ward, J. C. Correlations and spontaneous magnetization of the two-dimensional Ising model. J. Mathematical Phys. 4 (1963) 308-322.

[11] McCoy, B. and Wu, T. T. The Two-Dimensional Ising Model. Harvard University Press, 1973.

[12] Wu, T. Theory of Toeplitz Determinants and the Spin Correlations of the Two-Dimensional Ising Model. I. Phys. Rev. 149 (1966) 380-401.

[13] Kadanoff, L. Spin-spin correlations in the two-dimensional ising model. Nuovo Cimento (ser. 10) B 44 (1966) 276-305.

[14] Cheng, H. and Wu, T. Theory of Toeplitz Determinants and the Spin Correlations of the Two-Dimensional Ising Model. III. Phys. Rev. 164 (1967) 719-735.

[15] Baxter, R. J. Solvable eight-vertex model on an arbitrary planar lattice. Philos. Trans. Roy. Soc. London Ser. A 289 (1978) 315-346.

[16] Ghosh, R. K. and Shrock, R. E. Exact expressions for diagonal correlation functions in the $d=2$ Ising model. Phys. Rev. B (3) 30 (1984) 3790-3794. 
[17] Reyes Martínez, J. R. Correlation functions for the $Z$-invariant Ising model. Phys. Lett. A 227 (1997) 203-208.

[18] Jimbo, M. and Miwa, T. Studies on holonomic quantum fields. XVII. Proc. Japan Acad. Ser. A Math. Sci. 56 (1980) 405-410; 57 (1981) 347.

[19] McCoy, B. M., Tracy, C. A., and Wu, T. T. Painlevé functions of the third kind. J. Mathematical Phys. 18 (1977) 1058-1092.

[20] Boukraa, S., Hassani, S., Maillard, J.-M., McCoy, B. M., Orrick, W. P., and Zenine, N. Holonomy of the Ising model form factors. J. Phys. A 40 (2007) 75-111.

[21] McCoy, B. M. and Wu, T. T. Nonlinear partial difference equations for the two-spin correlation function of the two-dimensional Ising model. Nuclear Phys. B 180 (1981) 89115.

[22] Au-Yang, H. and Perk, J. H. H. Correlation functions and susceptibility in the $Z$-invariant Ising model. In MathPhys odyssey, 2001, volume 23 of Prog. Math. Phys., pages 23-48. Birkhäuser Boston, Boston, MA, 2002.

[23] McCoy, B. M., Assis, M., Boukraa, S., Hassani, S., Maillard, J.-M., Orrick, W. P., and Zenine, N. The saga of the Ising susceptibility. preprint arXiv:1003.0751 (2010).

[24] Boukraa, S., Hassani, S., Maillard, J.-M., McCoy, B. M., Weil, J.-A., and Zenine, N. Painlevé versus Fuchs. J. Phys. A 39 (2006) 12245-12263.

[25] Forrester, P. J. and Witte, N. S. Application of the $\tau$-function theory of Painlevé equations to random matrices: $\mathrm{P}_{\mathrm{VI}}$, the JUE, CyUE, cJUE and scaled limits. Nagoya Math. J. 174 (2004) 29-114.

[26] Painlevé, P. Sur les Equations Differentielles du Second Ordre et d'Ordre Superieur, dont l'Interable Generale est Uniforme. Acta Math. 25 (1902) 1-86.

[27] Gambier, B. Sur le Equations Differentielles du Second Ordre et du Primier Degré dont l'Integrale est a Points Critiques Fixes. Acta Math. 33 (1910) 1-55.

[28] Okamoto, K. Studies on the Painlevé equations. I. Sixth Painlevé equation $P_{\mathrm{VI}}$. Ann. Mat. Pura Appl. (4) 146 (1987) 337-381.

[29] Picard, E. Mémoire sur la théorie des functions algébriques de deux varables", Journal de Liouville. Journal de Liouville 5 (1889) 135-319.

[30] Mazzocco, M. Picard and Chazy solutions to the Painlevé VI equation. Math. Ann. 321 (2001) 157-195.

[31] Kitaev, A. V. and Korotkin, D. A. On solutions of the Schlesinger equations in terms of $\Theta$-functions. Internat. Math. Res. Notices (1998) 877-905.

[32] Brezhnev, Y. V. A $\tau$-function solution of the sixth Painlevé transcendent. Theor. Math. Phys. 161 (2009) 1616-1633.

[33] Mangazeev, V. V. Picard solution of Painlevé VI and related tau-functions. preprint arXiv:1002.2327, submitted to J. Phys. A.: Mathematical and Theoretical . 
[34] Whittaker, E. and Watson, G. A course of modern analysis. "Cambridge University Press", Cambridge, 1996.

[35] Forrester, P. J. and Witte, N. S. Discrete Painlevé equations for a class of $P_{\mathrm{VI}} \tau$-functions given as $\mathrm{U}(N)$ averages. Nonlinearity 18 (2005) 2061-2088.

[36] Bostan, A., Boukraa, S., Guttmann, A. J., Hassani, S., Jensen, I., Maillard, J.-M., and Zenine, N. High order Fuchsian equations for the square lattice Ising model: $\tilde{\chi}^{(5)}$. J. Phys. A 42 (2009) 275209, 32. 